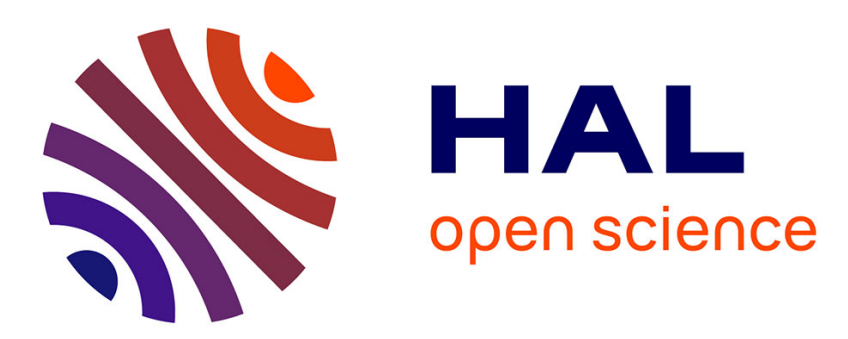

\title{
L'évolution des dissonances du syndicalisme français aux prises avec l'énergie électronucléaire et sa critique sociale
}

Mikael Chambru

\section{To cite this version:}

Mikael Chambru. L'évolution des dissonances du syndicalisme français aux prises avec l'énergie électronucléaire et sa critique sociale. Mouvements: des idées et des luttes, 2014, 80, pp.67-77. 10.3917/mouv.080.0067 . hal-01358141

\section{HAL Id: hal-01358141 \\ https://hal.science/hal-01358141}

Submitted on 16 Nov 2016

HAL is a multi-disciplinary open access archive for the deposit and dissemination of scientific research documents, whether they are published or not. The documents may come from teaching and research institutions in France or abroad, or from public or private research centers.
L'archive ouverte pluridisciplinaire HAL, est destinée au dépôt et à la diffusion de documents scientifiques de niveau recherche, publiés ou non, émanant des établissements d'enseignement et de recherche français ou étrangers, des laboratoires publics ou privés. 


\title{
L'ÉVOLUTION DES DISSONANCES DU SYNDICALISME FRANÇAIS AUX PRISES AVEC L'ÉNERGIE ÉLECTRONUCLÉAIRE ET SA CRITIQUE SOCIALE
} Mikaël Chambru

\author{
La Découverte | «Mouvements »
}

2014/4 nº 80 | pages 67 à 77

ISSN 1291-6412

ISBN 9782707183361

Article disponible en ligne à l'adresse :

http://www.cairn.info/revue-mouvements-2014-4-page-67.htm

\section{Pour citer cet article :}

Mikaël Chambru, « L'évolution des dissonances du syndicalisme français aux prises avec l'énergie électronucléaire et sa critique sociale », Mouvements 2014/4 ( $\left.n^{\circ} 80\right)$, p. 67-77.

DOI 10.3917/mouv.080.0067

Distribution électronique Cairn.info pour La Découverte.

(C) La Découverte. Tous droits réservés pour tous pays.

La reproduction ou représentation de cet article, notamment par photocopie, n'est autorisée que dans les limites des conditions générales d'utilisation du site ou, le cas échéant, des conditions générales de la licence souscrite par votre établissement. Toute autre reproduction ou représentation, en tout ou partie, sous quelque forme et de quelque manière que ce soit, est interdite sauf accord préalable et écrit de l'éditeur, en dehors des cas prévus par la législation en vigueur en France. Il est précisé que son stockage dans une base de données est également interdit. 


\section{L'évolution des dissonances du syndicalisme français aux prises avec l'énergie électronucléaire et sa critique sociale}

Après avoir retracé l'engagement des syndicats dans le processus de nucléarisation de la France, Mikaël Chambru propose d'étudier l'évolution des relations d'interdépendances entre les organisations syndicales et la mouvance antinucléaire. Tout d'abord, il décrypte la brèche introduite dans cet engagement par l'irruption du phénomène antinucléaire au tournant des années 1970. Ensuite, il retrace les dissonances provoquées au cours des décennies suivantes par les processus croisés de convergence et de discordance entre ces deux protagonistes contestataires. Enfin, il explore les potentialités contemporaines d'une convergence ouvrant la voie d'une transition vers une société postnucléaire.

$\mathrm{D}$ e décennies en décennies, la ligne de fracture entre syndicalisme et antinucléaire semble se perpétrer inéluctablement, selon les jeux de représentations liés au projet de construction d'une France technoscientifique. Comme si ces deux "visions du monde " étaient irréconciliables et s'imposaient aux individus s'engageant dans cette controverse sociotechnique engendrée par la technologie nucléaire. Nombre de militants, de journalistes et de citoyens partagent aujourd'hui cette vision, se positionnent vis-à-vis de celle-ci et participent sans cesse, de par leur prise de position publique et leur action, à la renouveler. En portant une attention particulière à l'historicité des interactions continues entre ces deux phénomènes contestataires, cet article vise au contraire à mettre en évidence, sans pour autant occulter les tensions engendrées, leurs moments de convergence et les potentialités ouvertes par ceuxci. En ce sens, il s'agit de repérer et d'analyser conjointement les déplacements de l'action protestataire syndicale et antinucléaire, des années 1960 aux années 2010. L'objectif est ici de montrer que l'engagement des
Par MikaËL

Chambru*
* Docteur en sciences de l'information et de la communication, université GrenobleAlpes, Groupe de recherche sur les enjeux de la communication. 
1. F. Chateauraynaud, "Le sens de l'irréversible. Chronique du nucléaire civil après Fukushima ", in Socio-informatique et argumentation, mai 2011.

2. M. Chambru, Communication, délibération et mouvements sociaux. L'espace public à l'épreuve du phénomène antinucléaire en France (1962-2012), Thèse de doctorat en Sciences de l'information et de la communication, université GrenobleAlpes, juin 2014.

3. G. Неснт, Le rayonnement de la France. Energie nucléaire et identité nationale après la seconde guerre mondiale, Paris, Éditions Amsterdam, 2014, p. 385-386.

4. Ibid., p. 170.

5. B. Christophe, "Les transformations des rapports entre sciences et société en France depuis la Seconde Guerre mondiale : un essai de synthèse ", in J. LE MAREC (dir.), Sciences, médias et société. Actes de colloque, Lyon, École normale supérieure Lettres et Sciences humaines, 2005, p. $19-20$. syndicats dans le processus de nucléarisation de la France est flexible, pluriel et négociable : les positions des organisations syndicales fluctuent, divergent, se rapprochent, se différencient, entre elles, entre les niveaux d'une même organisation, entre les différents lieux d'engagement, avec les mouvements sociaux critiquant le programme électronucléaire français, etc. Ces dissonances révèlent que des bifurcations sont réalisables dans les trajectoires protestataires, rendant ainsi possibles l'émergence d'un "point d'irréversibilité ${ }^{1}$ ", au sein de cette controverse sociotechnique. Cet article s'appuie sur un travail de recherche doctorale visant à analyser l'émergence, le déploiement et la résilience de cette dernière au sein de l'espace public sur une période de cinquante ans ${ }^{2}$.

\section{- L'engagement syndical dans le processus de nucléarisation}

Dès son lancement au lendemain de la Seconde Guerre mondiale, l'aventure nucléaire française est investie, par une logique de mobilisation visant au redressement du pays et à la construction d'une identité nationale positive. Le but est de restaurer, par le développement technique et l'enchevêtrement entre énergie nucléaire et identité nationale, la "francité de la nation " et ses " caractéristiques apparemment éternelles " que sont "le rayonnement, la gloire et la grandeur "3. Au cours des années 1950 et 1960, l'ensemble des acteurs sociaux institués participent de cette dynamique de restauration de la stature politique du pays sur le plan international et d'accélération de son redressement industriel. Les principales organisations syndicales, notamment la Confédération générale du travail (CGT) et la Confédération française démocratique du travail (CFDT), partagent ce choix. Elles sont par conséquent pleinement engagées en faveur de cette prouesse technique et scientifique, tout en s'efforçant de l'intégrer dans le combat du progrès social. Jugeant que "le monde sociotechnique en mutation exigeait que les frontières traditionnelles entre les classes soient transcendées ", les syndicats voient en effet " dans le savoir technique une occasion et un lieu de pouvoir "leur permettant d'" accéder à ce pouvoir ${ }^{4}$ ". En ce sens, ils s'accordent pour donner un rôle central aux ouvriers dans le développement du programme électronucléaire. Pour cela, les organisations syndicales tendent au cours de cette période à gommer leurs dissemblances dans le processus de nucléarisation de la France.

Ce choix consensuel des syndicats de présenter unanimement le recours à l'énergie électronucléaire comme un impératif de modernisation de la nation s'inscrit dans la conjoncture sociopolitique de la période de l'après-guerre. Celle-ci est effectivement marquée par une "surdétermination " de la présence de la science et de la technique dans l'espace public et par une "adhésion délégative " de larges pans de la société au paradigme modernisateur, dépassant la seule thématique du nucléaire 5 . Elle conduit alors les individus cherchant, au tournant des années 1970, à briser le consensus social dominant sur les bienfaits de l'atome civil à s'engager en marge de l'espace public institué et des organisations syndicales agissant en son sein. Leurs actions visent alors à publiciser les risques liés à la technologie nucléaire déjà pris en charge dans l'opacité 
par les autorités publiques en les soumettant à la discussion et au débat contradictoire, dans le but de les rendre visibles auprès du public et ensuite de mobiliser celui-ci pour qu'il participe à la contestation naissante. En ce sens, les mouvements sociaux transforment une question initialement qualifiée de technique en un enjeu politique porteur d'un choix collectif et global touchant aux orientations générales de la société. Cette politisation et cette mise en public de la menace nucléaire provoquent simultanément une redistribution partielle des jeux de représentations et d'arguments, des rapports de force, des jeux d'acteurs et des stratégies communicationnelles de l'ensemble des acteurs sociaux ${ }^{6}$. Bien qu'engagés de longue date dans le processus de nucléarisation de la France, les syndicats ne sont ni aveugles et ni sourds aux revendications des mouvements sociaux critiquant la technologie nucléaire. Ils cherchent ainsi rapidement à exercer une certaine influence sur les problématiques soulevées par la mouvance antinucléaire, d'autant plus que ces dernières interpellent le syndicalisme et son intérêt croissant au cours de cette période pour les questions environnementales ${ }^{7}$. Depuis la fin des années 1960, les missions constitutives de l'action syndicale s'articulent en effet progressivement, "dans les discours et dans les pratiques ", avec les enjeux environnementaux, notamment par le biais de la notion de cadre de vie au sein de la CFDT $^{8}$.

Dès lors, ces débordements protestataires antinucléaires introduisent progressivement une bifurcation dans l'angle des visions et des croyances jusqu'alors instituées. Elle se traduit, en situation, par le tissage de relations d'interdépendances et par l'amorce d'une convergence partielle entre ces deux protagonistes contestataires. Dès 1971, quelques militants de la CFDT participent ainsi aux premières manifestations antinucléaires organisées sur le territoire hexagonal ; avant d'être de plus en plus nombreux à s'interroger sur la signification sociale du programme électronucléaire puis à rejoindre, au cours des années suivantes, les différentes coalitions antinucléaires locales se constituant sur les territoires menacés par l'implantation d'un site électronucléaire. Cet engagement progressif de militants syndicaux fait alors resurgir la tension historiquement ancrée depuis deux décennies au sein de la CFDT, entre la frange jugeant la technique comme "incontrôlable et oppressive " et celle considérant son développement comme " [pouvant et devant] être modelé par des valeurs humaines ${ }^{9}$ ». En ce sens, l'irruption de la mouvance antinucléaire est génératrice de dynamiques ayant des retentissements sur l'ensemble des acteurs sociaux. Elle révèle l'hétérogénéité des visions et des enjeux au sein de la CFDT, en même temps qu'elle
6. M. Chambru, "La critique du régime technopolitique des sciences par la mouvance antinucléaire : un éclairage sur le concept d'espace public oppositionnel ", in Les enjeux de l'information et de la communication, 2014, à paraître.

\section{R. Mouriaux et C. Villaneuva, " Les syndicats français face à l'écologie, de 1972 à 1992 ", in Mots, numéro 29, juin 1994, p. $37-44$.}

\section{R. BÉCOT, "L'intervention syndicale de l'environnement dans la France des années 1960 ", in Vingtième siècle. Revue d'bistoire, numéro 113, 2012 . p. 171-177.}

\section{Bien qu'engagés de longue date dans le processus de nucléarisation de la France, les syndicats ne sont ni aveugles et ni sourds aux revendications des mouvements sociaux critiquant le technologie nucléaire.}

9. G. Неснт, Le rayonnement de la France, op. cit., p. 140. 
bouscule les rapports de forces internes relatifs aux sens et aux objectifs à donner au syndicat. La façade unanimiste de ce dernier, jusqu'alors articulée autour d'une participation active au processus de nucléarisation de la France conjointement à une critique du système capitaliste, de la science et de la technologie avec la proposition d'une société autogestionnaire, s'en retrouve ébranlée.

\section{- La convergence syndicale avec la mouvance antinucléaire}

En avril 1975, ces dissentiments finissent par provoquer une bifurcation officielle dans l'angle des visions de la CFDT. Celle-ci résulte aussi

\section{Ce positionnement ambigu résulte en fait d'un compromis entre les deux tendances s'opposant au sein du syndicat et renouvelant ici leurs postures : l'une est radicalement contre le nucléaire, l'autre l'admet sous condition et sous contrôle.} de l'évolution d'un second aspect de la conjoncture sociopolitique française : l'accélération du programme électronucléaire décidé en 1974 par le gouvernement, via le Plan Messmer. Ce dernier prévoit en effet la construction d'environ quatre-vingts centrales nucléaires jusqu'en 1985, pour ensuite atteindre un total de 170 installations en 2000. Percevant ces nouvelles modalités de réalisation du programme électronucléaire français comme " une sorte d'irréver-

10. M.-J. CARrieu, "La CFDT dans le débat sur le nucléaire ", in F. FAgnani et A. Nicolon (dir.), Nucléopolis. Matériaux pour l'analyse d'une société nucléaire, Grenoble, PUG, 1979, p. 152. sibilité rendant plus difficile la réalisation d'un autre modèle social ${ }^{10}$ ", la CFDT adopte à ce moment-là, et pour la première fois, une position ouvertement et résolument hostile vis-à-vis de celui-ci. Pour autant, elle ne s'oppose pas totalement et a priori à l'énergie nucléaire. Ce positionnement ambigu résulte en fait d'un compromis entre les deux tendances s'opposant au sein du syndicat et renouvelant ici leurs postures : l'une est radicalement contre le nucléaire, l'autre l'admet sous condition et sous contrôle. En pratique, il se traduit par la revendication d'un moratoire de trois ans du plan Messmer. Remettant désormais explicitement en cause le processus décisionnel prévalant jusqu'alors en matière atomique, l'objectif de la CFDT est de réduire au minimum la place du nucléaire dans la politique énergétique, tout en poursuivant son travail visant à renforcer la sûreté des installations électronucléaires et la sécurité des travailleurs. En jeu autour de cette question nucléaire, la mise en cause de l'idéologie du progrès et de la science.

Dans cette perspective, la CFDT établit pendant plusieurs années une collaboration avec la mouvance antinucléaire, et ce, même si les relations ne sont ni suivies ni structurées au niveau national. Sa posture non-strictement antinucléaire s'insère alors dans l'amas d'acteurs sociaux partiellement structuré/morcelé, aux contours imprécis, diffus et mouvants constituant la mouvance antinucléaire et au sein duquel se déploient des logiques d'acteurs, des temporalités, des enjeux et des tactiques hétéroclites. Celles-ci entrent parfois en résonance, parfois en tension avec 
les différentes positions des militants de la CFDT : opposition à un projet particulier, opposition à l'ensemble du programme gouvernemental ou opposition plus large à tout recours à l'énergie électronucléaire, etc. Ainsi, au sein de la mouvance antinucléaire comme au sein de la CFDT, ce n'est pas la référence dogmatique à une idéologie qui maintient la cohésion des individus, mais les applications technopolitiques qu'ils défient. En pratique, cet engagement syndical se traduit par l'organisation de débats publics, par la participation aux tentatives de structuration des coalitions régionales, par la diffusion de l'information, par la réalisation des performances protestataires, par la production de films cinématographiques, etc. Dans le milieu scientifique, il se matérialise également par une participation active au processus de politisation du nucléaire civil initié par plus de 4000 chercheurs signataires de l'“ Appel de scientifiques à propos du programme nucléaire français ". En s'appuyant sur leurs compétences professionnelles, l'objectif de ces scientifiques est de prendre position au sein de la controverse, en posant publiquement la question du risque nucléaire et en remettant en cause les liens institués entre nucléaire et intérêt national, entre progrès technique et progrès social $^{11}$. Dès lors, la CFDT joue un rôle déterminant dans la production de la contre-expertise antinucléaire, en lui apportant une caution et une argumentation scientifique indispensable pour appuyer et légitimer son action protestataire.

Dans le même temps, cet engagement de la confédération syndicale dans les dynamiques contestataires antinucléaires est sans cesse distancié, au sens où elle n'hésite pas à s'éloigner de la mouvance antinucléaire dès lors qu'elle juge la posture défendue par celle-ci non conforme à son positionnement. En 1977, par exemple, elle se retire au dernier moment de la manifestation du 31 juillet contre le surgénérateur Superphénix face à l'absence d'une action orientée et organisée sur des objectifs précis, et ce alors qu'elle avait dans un premier temps participé à la préparation de celle-ci et qu'elle avait pris une position hostile à ce type de réacteur dès 1973. Cet engagement distancié de la CDFT produit des tensions avec les composantes de la mouvance antinucléaire, mais aussi entre les différents militants du syndicat. Cette instabilité épargne par contre largement la CGT qui reste résolument hostile à la contestation antinucléaire, et ce, même si le syndicat s'oppose, dès 1974, aux modalités du programme gouvernemental inscrites dans le Plan Messmer. Considérant plus que jamais le nucléaire comme "participant d'un mouvement de croissance et de progrès forcément bénéfique pour la société ", la CGT renvoie dos à dos le gouvernement et la mouvance antinucléaire, dont l'" action insidieuse vise à discréditer la science, ils ne sont pas moins condamnables que le premier ${ }^{12}$ ". Au cours des années 1970 s'affirment donc des distinctions sensibles entre les différentes façons dont les syndicats conceptualisent les relations entre la technique et la politique, puis concrétisent leur participation à l'accélération du processus de nucléarisation de la France, et enfin envisagent le rôle du syndicat dans la société.
11. S. Topçu, "Nucléaire : de l'engagement 'savant' aux contreexpertises associatives ", in Natures sciences sociétés, numéro 14 , 2006, p. 252-253.

12. M.-J. CARRIEU, "La position de la CGT dans le débat sur le nucléaire ", in Nucléopolis. Matériaux pour l'analyse d'une société nucléaire (dir. Francis Fagnani F. et al.), Grenoble, PUG, 1979, p. 156-159. 


\section{- L'emprise des dissonances syndicales et leurs retentissements}

Entre 1975 et 1981, ces dissonances syndicales sont surmontées en Basse-Normandie, avec l'établissement d'une alliance synchronique, entre l'Union régionale (UR) CFDT et le Comité de réflexion, d'information et de lutte antinucléaire (Crilan). Cette convergence est favorisée par le multipositionnement ancien de certains militants antinucléaires dans le champ syndical et par les liens affinitaires noués avec des acteurs éminents de celui-ci. De bonnes relations s'éta-

Assez rapidement l'unité d'action
se fissure et la CGT de Cherbourg
prend ses distances avec la
contestation, rappelée à l'ordre en
ce sens par la CGT, celle-ci étant
favorable, au niveau national,
au retraitement des déchets
radioactifs étrangers à la Hague.

blissent entre les deux entités qui parviennent à combiner habilement, via la dénonciation du risque nucléaire, la critique syndicale et la critique antinucléaire. En ce sens, ils mettent en pratique et élargissent le rapprochement théorique déjà initié entre la CFDT et la mouvance antinucléaire, autour de la problématique des risques et des conditions de travail pour les salariés de l'industrie électronucléaire. Cette action commune
13. A. Touraine et al., $L a$ prophétie antinucléaire, Paris, Seuil, 1984, p. $122-133$. s'articule en effet autour de l'opposition conjointe à la privatisation et à l'extension de l'usine de retraitement de la Hague, mais aussi autour de la défense conjointe des conditions de travail des salariés du nucléaire et de la sûreté des installations électronucléaires. Elle sera notamment marquée, à l'automne 1976, par la grève de trois mois des salariés de l'usine initiée par l'UR-CFDT et soutenue par le Crilan, ainsi que par les Assises du nucléaire de Cherbourg. Cette convergence n'en est pas moins instable, puisqu'elle s'inscrit dans une conjoncture nationale de plus en plus marquée par une "séparation " entre les revendications syndicales et les positions antinucléaires "peu portées par la base " de la CFDT ${ }^{13}$.

Toutefois, elle se prolonge et s'amplifie localement, à partir de 1978, à travers le mouvement d'opposition aux transports de déchets radioactifs étrangers devant être "retraités " à l'usine de la Hague. De nouveaux protagonistes rejoignent la mobilisation, notamment la CGT de Cherbourg. Le 16 décembre, elle défile ainsi une première fois avec les militants antinucléaires et ceux de la CFDT derrière une banderole " Non aux déchets étrangers ". Le 22 janvier 1979, le cargo transportant les premiers combustibles irradiés japonais vers La Hague arrive au port de Cherbourg. En réaction, l'UR-CFDT lance un mot d'ordre de grève générale, des préavis sont déposés dans plusieurs entreprises, plusieurs sections professionnelles locales de la CGT se joignent au mouvement : 7000 personnes tentent de s'opposer à l'arrivée des déchets japonais, en vain. Assez rapidement ensuite, l'unité d'action se fissure et la CGT de Cherbourg prend ses distances avec la contestation, rappelée à l'ordre en ce sens par la CGT, celle-ci étant favorable, au niveau national, au retraitement des déchets radioactifs étrangers à la Hague. En agissant ainsi, la 
CGT stoppe brutalement la dissonance émergente : difficile en effet d'être un ardent défenseur de l'énergie électronucléaire tout en étant contre l'usine de la Hague qui est la pierre angulaire du programme électronucléaire français. Bien que cet engagement local d'une organisation historiquement marquée par une adhésion idéologique au nucléaire dans une des dynamiques antinucléaires reste partiel et circonscrit temporellement, il montre toutefois que des brisures sont toujours possibles dans les trajectoires et dans l'angle des visions syndicales dès lors que s'ouvre une opportunité dans la conjoncture sociopolitique.

Au tournant des années 1980 s'amorce progressivement une désunion entre la CFDT et la mouvance antinucléaire sur fond de divergences stratégiques. En cause, l'évolution de la conjoncture sociopolitique avec l'arrivée de la gauche au pouvoir en 1981. Celle-ci renforce les dissonances du syndicat pour qui les contradictions sont de plus en plus difficiles à tenir et à assumer. La CFDT participe en effet activement au processus d'" hyper-institutionnalisation " de la critique antinucléaire orchestrée par les pouvoirs publics au cours de cette période ${ }^{14}$, voyant dans celui-ci une opportunité de concrétiser son engagement ancien sur la sûreté des installations pour les populations et pour la sécurité des travailleurs. À l'inverse, une frange significative de la mouvance antinucléaire s'y refuse, jugeant que le but de cette entreprise est de canaliser et de résorber les débordements contestataires par la création de nouveaux espaces institutionnels et de nouveaux espaces de concertation. Ensuite, une seconde évolution conjoncturelle vient renforcer les dissonances de la CFDT : le déplacement progressif de l'action antinucléaire de l'opposition à la construction de nouvelles centrales nucléaires vers la revendication de fermeture des installations déjà existantes. Résonnant autour des enjeux de court terme, le syndicat s'engage alors pour la défense de l'emploi industriel sur les sites menacés, accentuant ainsi encore un peu plus ses discordances avec la mouvance antinucléaire. Dans les années quatrevingt-dix, la CFDT ne s'associe par exemple pas avec cette dernière pour réclamer l'arrêt du surgénérateur de Superphénix, alors même qu'elle refusait qu'il soit construit quinze ans plutôt.

\section{- L'ouverture d'une brèche dans l'angle des visions syndicales}

Au printemps 1998, la thématique du risque nucléaire resurgit dans le champ syndical au prisme du transport des déchets radioactifs. S'appuyant sur une enquête menée pendant plusieurs mois par le cabinet d'expertise Wise-Paris, Libération révèle, dans son édition du 6 mai, que des transports de combustibles irradiés, en provenance des centrales et à destination de l'usine de la Hague, sont contaminés et traversent la France depuis plusieurs années, mais aussi que les sociétés en charge de ces derniers sont parfaitement au courant de ces problèmes de contamination et qu'elles ont délibérément fait le choix de ne pas les rendre publics. Le risque sanitaire est double : d'une part, les gens peuvent être contaminés par les particules radioactives se répandant dans l'environnement le long des voies de chemin de fer ; et d'autre part, des cheminots ont reçu
14. S. TOPÇ, $L a$

France nucléaire.

L'art de gouverner une technologie contestée, Paris, Seuil, 2013, p. 121-133. 
15. M. SCHNEIDER, "La saga des transports contaminés. Un récit personnel ", in Investigation plutonium, 6-7, 1998, p. 8-9.

16. F. PACCOU, La fédération des syndicats SUD-rail (1996-2005), Nancy, IRENEE, 2006, p. $18-23$. des doses jusqu'à trente fois supérieures à la norme. Le jour même, plusieurs syndicats de la SNCF exigent l'application de leur " droit de retrait ", via le Comité d'hygiène, de sécurité et des conditions de travail (CHSCT). Parmi eux, la CGT réclame que les cheminots confrontés aux transports contaminés disposent de dosimètres et subissent des examens médicaux réguliers au même titre que les travailleurs du nucléaire, reprenant ainsi à son compte une veille revendication de la CFDT. En réponse, la direction de la SNCF décide de stopper temporairement ces transports pendant quelques semaines, avant que ceux-ci ne reprennent au début de l'été, une fois l'emballement médiatique et politique estompé, et après que la Direction de la sûreté des installations nucléaires (DSIN) - l'ancêtre de l'Autorité de sûreté nucléaire (ASN) - ait conclu à "l'absence de tout risque sanitaire ${ }^{15}$ ".

Au moment où survient cette crise, Sud-Rail - le dernier né des syndicats à la SNCF suite à une scission au sein de la CFDT en 1996 - est dans une dynamique de construction et de développement ${ }^{16}$. Cherchant à se distinguer des autres organisations syndicales et sensibles à la problématique du nucléaire de part l'engagement de certains de ses militants, il décide d'investiguer au sein de la SNCF sur ces dangers pour les cheminots. Pour cela, Sud-Rail fait appel à la Commission de recherche et d'information indépendante sur la radioactivité (Criirad), laboratoire associatif créé en 1986 et reconnu pour sa contre-expertise scientifique. Ses analyses confirment les inquiétudes du syndicat : au-delà du problème de la contamination des wagons, le combustible irradié émet des rayonnements posant des problèmes en cas d'exposition. En s'appuyant sur ces résultats, Sud-Rail s'active pendant un an à faire adopter des mesures de protection des cheminots via le CHSCT, sans grand résultat. Parallèlement, un débat s'instaure au sein du syndicat en 1999 : faut-il réclamer la qualité de travailleur du nucléaire pour les cheminots travaillant sur les transports de déchets radioactifs ? Après plusieurs mois de discussions, la réponse est non, les syndicalistes refusant d'accepter un taux irradiation supérieure pour obtenir ce statut. La dose maximale admissible est en effet d'un millisievert (mSv) par an pour la population et de $100 \mathrm{mSv}$ sur cinq ans, avec un maximum de $50 \mathrm{mSv}$ en une seule année, pour les salariés du nucléaire. À ce moment-là, Sud-Rail n'inscrit pas son action dans une perspective antinucléaire, et rare sont encore les militants du syndicat à défendre une telle posture. Toutefois, ce débat interne initie chez les cheminots, à partir de leur réalité vécue, un début de réflexion sur le nucléaire, ses atouts et ses dangers.

En 1999 toujours, Sud-Rail établit son premier contact avec la mouvance antinucléaire, à l'occasion de la reprise des transports de déchets radioactifs allemands suspendus un an plutôt. Ce transit international est jugé illégitime par le syndicat, car il fait prendre des risques aux cheminots, non pas pour faire fonctionner les centrales nucléaires françaises, mais pour faire un "commerce des déchets nucléaires". Cette prise de position coïncide alors avec le choix du Réseau sortir du nucléaire (RSN) de faire de l'opposition à ces transports de déchets radioactifs un des 
axes de sa stratégie d'action visant à publiciser le risque nucléaire. Ce qui intéresse alors les militants antinucléaires, ce sont les trajets et les horaires des convois atomiques dont disposent les syndicalistes de la SNCF, dans le but de préparer leur performance protestataire et ainsi d'agir le plus efficacement possible. Une coopération informelle se noue pendant plusieurs années contre ces transits internationaux, Sud-Rail distillant diverses informations sur les convois atomiques au RSN, tout en lui apportant son expertise sur le sujet. Parallèlement, un débat de longue haleine s'engage au sein du syndicat sur le nucléaire. En 2003, il aboutit à la décision de s'opposer aux transports ferroviaires de déchets radioactifs. Il faut ensuite attendre 2009 pour que Sud-Rail prenne explicitement un virage antinucléaire, mettant ainsi fin à une longue période de dissonances. Dès lors, le syndicat participe activement aux actions contre les transports de déchets radioactifs, apportant ainsi une légitimité supplémentaire à la mouvance antinucléaire. Cette convergence se concrétise notamment en novembre 2011 par la participation active de Sud-Rail à la mobilisation du collectif Valognes Stop Castor visant à perturber le départ d'un train de déchets radioactifs pour l'Allemagne ${ }^{17}$.

\section{- Les potentialités contemporaines d'une convergence}

La trajectoire de Sud-Rail est intéressante à plusieurs égards dès lors que l'on s'intéresse aux potentialités contemporaines d'une convergence entre syndicalisme et antinucléarisme. Premièrement, elle montre que le renversement des rapports et des croyances jusqu'alors institués au sein du syndicat prend inévitablement du temps, nécessite de nombreuses discussions et se déroule par étapes successives. Il faut d'abord que cette question du nucléaire soit perçue comme étant porteuse de sens, nécessitant pour cela l'engagement précoce d'un certain nombre de militants, en discordance avec la position officielle du syndicat. Il faut ensuite parvenir à concilier, non sans difficulté, la revendication d'une sortie du nucléaire avec la raison d'être du syndicat qu'est la défense des intérêts des salariés. Cela nécessite, d'une part, une articulation des enjeux de court terme et ceux de plus long terme, et d'autre part le dépassement du dilemme institué de l'" emploi contre l'environnement "dans le champ syndical. En jeu, la prise de conscience que la question du nucléaire est un enjeu politique porteur d'un choix collectif et global touchant aux orientations générales de la société et qu'en ce sens elle ne concerne pas uniquement les seuls intérêts des cheminots. Deuxièmement, la trajectoire de Sud-Rail révèle que ce renversement ne résulte pas d'une adhésion idéologique a priori, mais d'un choix pragmatique s'opérant progressivement en situation en fonction de la logique
17. M. Chambru, "Valognes Stop Castor, continuité ou rupture des pratiques militantes antinucléaires?", in Revue des Livres, 5 , 2012, p. 74-79.

\section{Il faut ensuite parvenir à concilier, non sans difficulté, la revendication d'une sortie du nucléaire avec la raison d'être du syndicat qu'est la défense des intérêts des salariés.}


18. M. Chambru, "L'espace public à l'épreuve du phénomène antinucléaire en France (1962-2012)", in ESSACHESS - Journal for Communication Studies, 2014, p. 39-40. d'action du syndicat, des contraintes et des opportunités conjoncturelles. Il est en effet le produit d'interactions situées, entre un contexte relationnel et un contexte sociopolitique marqué par l'irruption d'un événement inattendu provoquant un basculement des enjeux et une redistribution partielle des jeux et des stratégies d'acteurs. La thématique du transport des déchets radioactifs et du risque nucléaire est en ce sens porteuse d'enjeux communicationnels cruciaux dans la dynamique de construction et de développement d'un syndicat récemment créé dans un espace syndical déjà fortement concurrentiel.

Cette convergence réussie entre Sud-Rail et la mouvance antinucléaire donne également à voir la nécessité de surmonter la disjonction des valeurs culturelles instituées au sein de ces deux phénomènes protestataires. Cette exigence est par exemple visible à travers la question de la libéralisation du marché de l'électricité. D’un côté, une frange de la mouvance antinucléaire voit dans cette ouverture du marché à la concurrence une opportunité permettant d'ébranler et d'affaiblir la toute-puissance du programme électronucléaire. Ils créent ainsi, en 2007, la Société coopérative d'intérêt collectif Enercoop, dans le but de mettre en ouvre et d'expérimenter d'autres modèles énergétiques incarnant l'alternative qu'ils défendent. De l'autre, une autre frange de la mouvance antinucléaire juge illusoire de croire que la privatisation et le recours à l'économie solidaire peuvent suffire à remettre en cause la politique énergétique française. Reste que cette initiative cherche à combler les manques et à étendre les prérogatives des services publics, tout en permettant aux choix énergétiques d'être intégrés dans l'espace des choix démocratiques ${ }^{18}$. En ce sens, le RSN adopte, via sa campagne "Pour un vrai service public de l'électricité sans nucléaire ", la posture suivante : défense du service public de l'énergie conjointement à sa démocratisation, décentralisation, déconcentration et dénucléarisation. Le but est alors d'identifier et de définir des enjeux communs entre les différentes composantes de la mouvance antinucléaire, mais aussi avec les syndicats et les syndicalistes - y compris ceux déjà ralliés à la cause antinucléaire - ceux-ci étant des ardeurs défenseurs du service public s'opposant à cette privatisation.

Ainsi, la fabrication de valeurs culturelles communes résulte aussi de la capacité de la mouvance antinucléaire à s'adresser aux syndicats en n'opposant pas impératif écologique et impératif social... avec la difficulté supplémentaire que cette conciliation est elle-même source de tensions et d'enjeux au sein de celle-ci, du fait de son caractère composite et de la pluralité de manières d'investir et d'envisager la critique sociale du programme électronucléaire. Là encore, il s'agit d'un processus en perpétuelle 
construction et s'inscrivant dans le temps long. À ce jour, seule l'Union syndicale solidaire - à laquelle adhère Sud-Rail - réclame une sortie du nucléaire, la CGT et la CFDT jugeant cette dernière toujours irréalisable. Le récent débat national sur la transition énergétique rappelle que les modalités argumentatives de ces dernières convergent davantage avec les organisations patronales et les entreprises du secteur électrique qu'avec la mouvance antinucléaire. Une situation que ne manque pas de relever et de critiquer cette dernière, quitte à ce que cela renforce les discordances et amenuise les potentielles convergences. Ces deux organisations syndicales s'accordent en effet, d'une part, pour défendre un mix-énergétique, certes avec une part plus ou moins importante de l'énergie électronucléaire entre les deux ; et d'autre part, pour défendre l'emploi industriel dès lors que celui-ci est menacé par les décisions gouvernementales de fermer des centrales nucléaires, là où la mouvance antinucléaire met en avant qu'une sortie du nucléaire peut se solder par un solde positif en termes d'emploi. Elles divergent par contre quant à la relance du programme électronucléaire via la construction de nouveaux types de réacteurs (EPR, Iter, Astrid) : la CGT en est une ardente promotrice, tandis que la CFDT s'y oppose, tout comme la mouvance antinucléaire.

\section{- Conclusion}

Certes des désaccords sensibles existent et persistent entre les syndicats et les mouvements sociaux, mais l'analyse conjointe des déplacements de l'action protestataire syndicale et antinucléaire sur le temps long montre que ces deux "visions du monde " ne s'opposent pas inéluctablement. L'histoire sociale est en effet marquée par l'établissement partiel, temporaire et localisé de convergences d'analyse, de revendications et d'actions entre ces deux phénomènes contestataires au sein de l'espace public. Aujourd'hui, elles restent à (re)construire autour des problématiques actuelles du risque nucléaire. Des syndicalistes et des militants antinucléaires sont déjà engagés dans cette voie, avec comme objectif d'ouvrir la voie d'une transition vers une société postnucléaire. En jeu également dans cette perspective, la place d'un syndicalisme déclinant au sein de l'espace des mouvements sociaux et plus largement au sein de la société française. Alors même qu'il a su "s'instituer comme composante " de celle-ci de par ses revendications universalisantes sur la protection sociale, les organisations syndicales restent tiraillées entre le "rôle de partenaire social " et celui de "façonneur de valeurs sociétales, porteuses de solidarités générales " dès lors qu'il est question des thématiques des risques, de l'environnement, des sciences et des techniques ${ }^{19}$. Une bifurcation de leur angle des visions est toutefois possible, en témoigne la récente prise de position de la CGT équipement d'Alsace en faveur de la fermeture de la centrale nucléaire de Fessenheim.
19. P. Chaskiel, "Du syndicalisme à la société civile : le 'risque industriel' comme frontière sociale ", in Actes du colloque international: Les cent ans de la charte d'Amiens, Amiens, Université Jules Vernes, 2006, p. 3-8. 